\title{
Preservation of electrophysiological functional connectivity after partial corpus callosotomy: case report
}

\author{
Kaitlyn Casimo, BA, ${ }^{1,2}$ Fabio Grassia, MD, ${ }^{3}$ Sandra L. Poliachik, PhD, ${ }^{3-5}$ Edward Novotny, MD, ${ }^{6,7}$ \\ Andrew Poliakov, PhD, ${ }^{3,4}$ and Jeffrey G. Ojemann, MD ${ }^{1,2,8}$

\begin{abstract}
${ }^{1}$ Graduate Program in Neuroscience and ${ }^{2}$ Center for Sensorimotor Neural Engineering, University of Washington, Seattle, Washington; ${ }^{3}$ Department of Neurosurgery, University of Milan, San Gerardo Hospital, Monza, Italy; and ${ }^{4}$ Department of Radiology, ${ }^{5}$ Center for Clinical and Translational Research, ${ }^{6}$ Department of Neurology, ${ }^{7}$ Center for Integrated Brain Research, and ${ }^{8}$ Department of Neurosurgery, Seattle Children's Hospital, Seattle, Washington
\end{abstract}

Prior studies of functional connectivity following callosotomy have disagreed in the observed effects on interhemispheric functional connectivity. These connectivity studies, in multiple electrophysiological methods and functional MRI, have found conflicting reductions in connectivity or patterns resembling typical individuals. The authors examined a case of partial anterior corpus callosum connection, where pairs of bilateral electrocorticographic electrodes had been placed over homologous regions in the left and right hemispheres. They sorted electrode pairs by whether their direct corpus callosum connection had been disconnected or preserved using diffusion tensor imaging and native anatomical MRI, and they estimated functional connectivity between pairs of electrodes over homologous regions using phase-locking value. They found no significant differences in any frequency band between pairs of electrodes that had their corpus callosum connection disconnected and those that had an intact connection. The authors' results may imply that the corpus callosum is not an obligatory mediator of connectivity between homologous sites in opposite hemispheres. This interhemispheric synchronization may also be linked to disruption of seizure activity.

https://thejns.org/doi/abs/10.3171/2018.2.PEDS17549

KEYWORDS functional connectivity; corpus callosum; partial corpus callosotomy; electrophysiology; electrocorticography; epilepsy

$\mathrm{F}$ UnCTIONAL connectivity describes coincident activity between brain regions. This may be the result of direct synchrony between brain regions, or of a common input similarly influencing multiple brain regions. It may support synaptic formation and maintenance, or provide a mechanism for cognitive integration and information transfer. ${ }^{11,27}$ The corpus callosum provides direct anatomical connections mostly between homologous regions, but its role in maintaining functional connectivity has not been fully established. Homologous sites in opposite hemispheres are generally robustly synchronized, which can be observed with both functional MRI (fMRI) and electrophysiological methods. ${ }^{12,13,23-25,31}$ Synchrony of apparently spontaneous origin exists at "rest," independent of any stimuli. ${ }^{32}$

Corpus callosotomy is performed to reduce the severity of drug-resistant epilepsy, particularly of drop attacks. The corpus callosum may be completely disconnected, or partially disconnected in the anterior or posterior portion. ${ }^{16}$ Approximately $75 \%$ of patients experience a reduction in frequency or severity of seizures after anterior callosotomy. ${ }^{26}$

After partial or complete disconnection of the corpus callosum, patients experience a reduction in cognitive integration, especially for language and visuospatial processing, which are particularly lateralized functions. ${ }^{3}$ Complete disconnection generally results in acute disruption of interhemispheric synchrony between homologous sites, both shortly after the procedure ${ }^{1,6,21,32}$ and during the course of the procedure itself, as measured with intraoperative scalp electroencephalography $(\mathrm{EEG})^{14}$ and with electrocorticography (ECoG). ${ }^{21}$

ABBREVIATIONS amHG = amplitude modulation of the high gamma band; BOLD = blood oxygen level-dependent; $\mathrm{DTI}=$ diffusion tensor imaging; $E C$ C $=$ electrocorticography; EEG = electroencephalography; fMRI = functional MRI; HG = high gamma; PLV = phase-locking value; VNS = vagus nerve stimulator.

SUBMITTED October 9, 2017. ACCEPTED February 13, 2018.

INCLUDE WHEN CITING Published online May 18, 2018; DOI: 10.3171/2018.2.PEDS17549. 
In a recent study, fMRI measures of interhemispheric synchrony following callosotomy were significantly reduced between homologous sites 7 days after surgery; synchrony rebounded by 28 days after surgery in individuals with partial disconnection, and remained depressed in those with complete disconnection. ${ }^{32} \mathrm{~A}$ case report of a 73-year-old patient showed near-normal interhemispheric blood oxygen level-dependent (BOLD) synchrony measured 40 years after complete commissurotomy including corpus callosum disconnection. ${ }^{30}$ In nonhuman primates 8 months after disconnection, partial callosotomy resulted in only moderate reduction in connectivity between the disconnected homologous regions, while increased connectivity arose in regions connected by the preserved portion of the corpus callosum. ${ }^{15}$

In most studies of agenesis of the corpus callosum, interhemispheric synchrony is preserved or nearly preserved compared to that of typical individuals. ${ }^{8,29,30}$ However, others have found little functional connectivity between hemispheres, despite near-typical connectivity within each hemisphere ${ }^{19,20}$ or found that both interhemispheric and intrahemispheric BOLD connectivity were disrupted, especially in regions involved in complex cognitive processing. ${ }^{4}$ In both agenesis of the corpus callosum and surgical disconnection, a variety of etiologies and comorbid developmental abnormalities contribute to a considerable heterogeneity, which may explain some of these discrepancies.

Here we present data from a case study in which the patient received a partial disconnection of the corpus callosum and simultaneous implantation of bilateral ECoG electrodes. Our finding of preserved connectivity in disconnected homologous pairs questions the model of direct callosal connections mediate connectivity, as proposed by Johnston et al. ${ }^{6}$

\section{Case Report}

Patient

A 17-year-old boy with intractable epilepsy underwent surgery to partially sever the corpus callosum.

\section{History}

Seizures developed at age 5 and were characterized by behavioral arrests or motor events and were frequently generalized. Neuropsychological testing identified significant deficits in executive function and slow processing speed. Scalp EEG revealed bilateral and diffuse frontal lobe changes. Seizures were not controlled by 11 different medications, a vagus nerve stimulator (VNS), or the ketogenic diet. Four years prior to the corpus callosum procedure, a VNS had been placed. He continued to have daily events and was admitted for bilateral frontal (lateral and medial) placement of subdural electrodes.

\section{Operations}

A partial corpus callosotomy was performed to attempt to limit seizure spread to assist in lateralization of the events. He underwent two surgeries: first, an anterior callosotomy and ECoG electrode implantation; and second, due to a lack of lateralizing information, removal of the ECoG electrodes and extension of the callosotomy. The
VNS was turned off for the time between surgeries, but his home anticonvulsant medications and schedule were not altered during his hospitalization. All data are from electrodes implanted at the time of the first operation.

\section{Data Acquisition}

Prior to the first surgery the patient underwent anatomical MRI and diffusion tensor imaging (DTI) (DTI: 20 directions, TR $4.33 \mathrm{sec}$; TE $105 \mathrm{msec}$, flip angle $90^{\circ}$, slice thickness $4 \mathrm{~mm}$, in-plane resolution $1.56 \times 1.56 \mathrm{~mm}$; sagittal T1-weighted MRI: T1-weighted MPRAGE resolution $1.3 \times 1.3 \mathrm{~mm}, 1 \mathrm{~mm}$ slice thickness). A 1.5-T MR imager was used instead of a 3-T system due to the presence of the VNS.

In the first surgery, the anterior portion of the corpus callosum was disconnected and 4 strips of ECoG electrodes were placed on homologous frontal areas of each hemisphere (Fig. 1A). A high-resolution CT scan was acquired to localize the electrodes. The electrodes were in place for 1 week, during which time the patient was found to have multifocal, bilateral seizure onset primarily originating in both frontopolar regions, with additional interictal activity from bilateral frontopolar, lateral frontal, and frontal midline regions. No electrophysiological data were recorded before the corpus callosum was cut in the first surgery, nor after the disconnection was extended in the second surgery.

We identified a putative resting state in the clinical ECoG recordings from day 7 after initial disconnection, shortly before the second surgery. An 8-minute restingstate segment when the patient was not engaged with any particular task and not asleep was extracted from clinical recordings.

\section{Data Processing}

We performed tractography of the corpus callosum in DSI Studio (http://dsi-studio.labsolver.org/) to determine which pairs of electrodes were located over cortical regions with severed or preserved direct corpus callosum connections. We outlined the corpus callosum in the midsagittal view on the T1-weighted image and tracked the fibers at a threshold of 0.15 . We used a relatively low threshold to compensate for the low resolution of the DTI images.

A landmark (Fig. 1A) generated by the Image Guided Surgery system indicated the extent of callosotomy ${ }^{18}$ and was coregistered with preoperative structural MR images using BioImage Suite. ${ }^{17}$ This landmark of the extent of the corpus callosum disconnection was used to divide the DTI tracts (Fig. 1B). Because the ECoG electrodes were in place during the entire period between partial disconnection in the first procedure and the extension of the disconnection in the second procedure, no MR image was obtained. Instead, ECoG electrode positions were identified as previously described, using BioImage Suite to coregister preoperative MR images and postoperative CT scans. ${ }^{2,17}$ We then labeled the pairs of electrodes as still connected by corpus callosum ("preserved"), no longer connected ("separated"), or ambiguous ("indeterminate") (Fig. 1).

All electrophysiological analyses were performed in Matlab (MathWorks). Preprocessing included the manual 

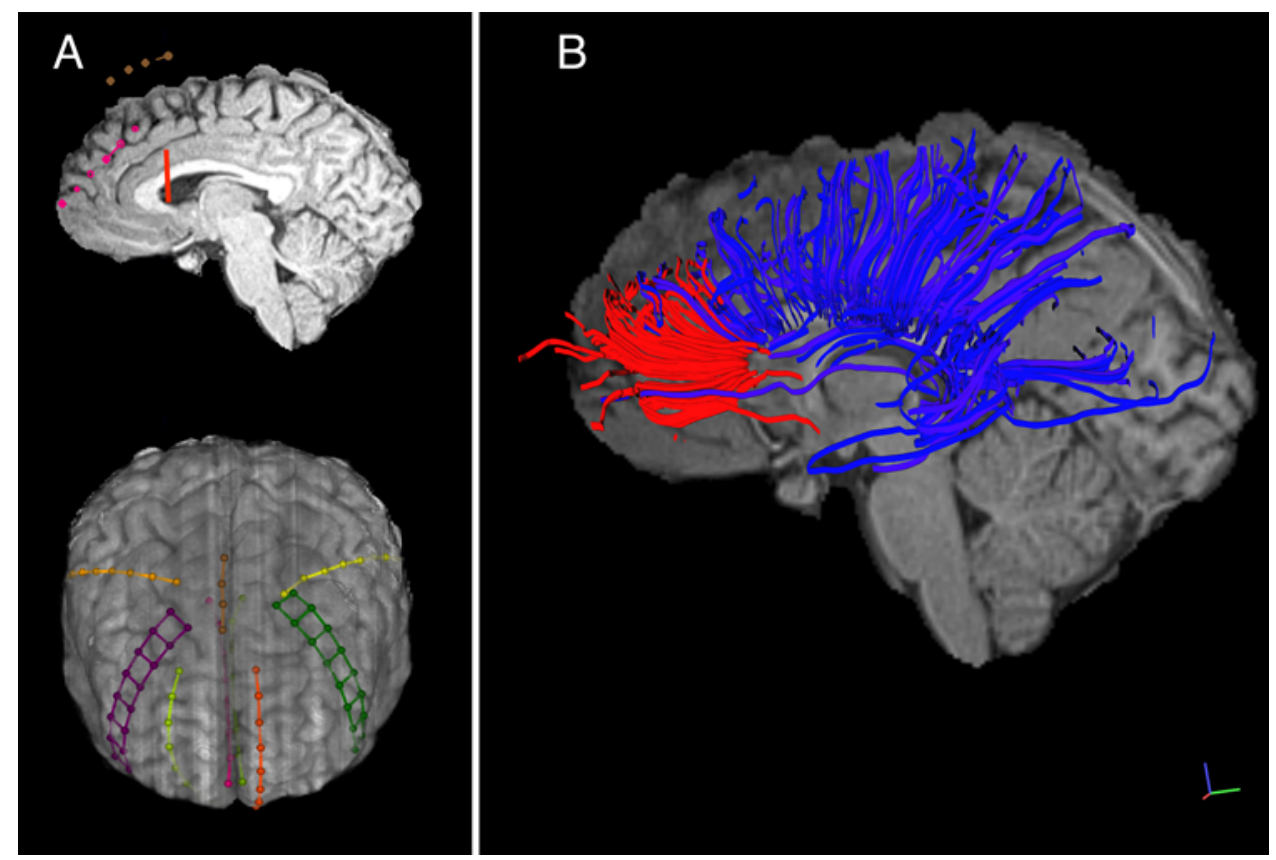

FIG. 1. A: High-resolution native T1-weighted image with locations of electrodes on the patient's own brain, which were placed over homologous regions. Red vertical line indicates the posterior extent of the first procedure severing part of the corpus callosum. Electrophysiological recordings were obtained exclusively at this stage of separation between hemispheres. B: Tracts traced using DTI scans overlaid on native T1-weighted MR images. Red tracts were separated and blue tracts were preserved. Based on these images, we divided the pairs of electrodes into 3 categories: 1) still directly connected via the corpus callosum (referred to as "preserved"; $n=12$ pairs); 2 ) no longer directly connected via the corpus callosum (referred to as "separated"; $n=23$ pairs); and 3 ) too close to the location of the cut to definitively determine (referred to as "indeterminate"; $n=9$ pairs). Indeterminate pairs were excluded from figures separating preserved and separated pairs (i.e., Figs. 2 and 3).

removal of nonphysiological artifacts, a common average reference across all electrodes, and a notch filter at 60, 120, and $180 \mathrm{~Hz}$. Interictal activity was avoided when selecting the resting-state period used from the extended clinical recording.

We band-pass filtered the signals at each frequency band of interest (delta, $0-4 \mathrm{~Hz}$; theta, 4-8 Hz; alpha, 8-12 $\mathrm{Hz}$; beta, 12-18 Hz; high gamma [HG], 70-200 Hz) using a zero-phase shift, fourth-order Butterworth filter. We calculated the amplitude modulation of the high gamma band (amHG), as previously described, using a secondary fourth-order Butterworth filter applied to the HG amplitude envelope. ${ }^{2,7,10}$ amHG connectivity is thought to uniquely represent resting-state network properties. ${ }^{2,9,10}$ We then estimated the instantaneous phase for all channels for each frequency band (including amHG) using a Hilbert transform.

\section{Connectivity Measures and Statistics}

We calculated pairwise phase-locking value (PLV) in each frequency band for each pair of equivalent electrodes located in opposite hemispheres. PLV measures the consistency in phase difference between a pair of signals, and it provides an estimate of synchrony between two time series. This may be interpreted as strength of functional integration between the brain regions where the signals were recorded. PLV was calculated across the full time series, using the following formula:

$$
\mathrm{PLV}=\Sigma \mathrm{e}^{\mathrm{i} * \Delta \theta}
$$

where $\Delta \theta$ is the instantaneous difference in phase between the two signals. ${ }^{11}$

We sorted the connectivity values between pairs of electrodes using their previously determined postsurgical corpus callosum status. Electrodes of indeterminate status were excluded from statistical analysis. We then performed a Wilcoxon rank-sum test comparing PLV between preserved and separated pairs. We repeated this comparison for each of the 7 frequency bands of interest.

\section{Results}

PLVs for preserved and separated electrode pairs in each frequency band of interest are shown in Fig. 2. There was no significant difference in connectivity strength between preserved and separated electrode pairs in any frequency band of interest (Wilcoxon rank-sum tests: all p > 0.05). There are variations in the median and spread in connectivity strength between the hemispheres in the different frequency bands, but there were no significant differences between preserved and separated pairs in each band. In particular, HG and amHG both had a higher median and a larger spread in PLVs than the other frequency bands.

Because the corpus callosum disconnection status for each electrode pair is an estimate based on low-resolution DTI tractography, we also investigated the relationship between PLV and anterior-posterior electrode position (Fig. 3). Independent of our DTI assessment of corpus callosum status, further anterior electrodes are more likely to have had their corpus callosum connection severed. There was 

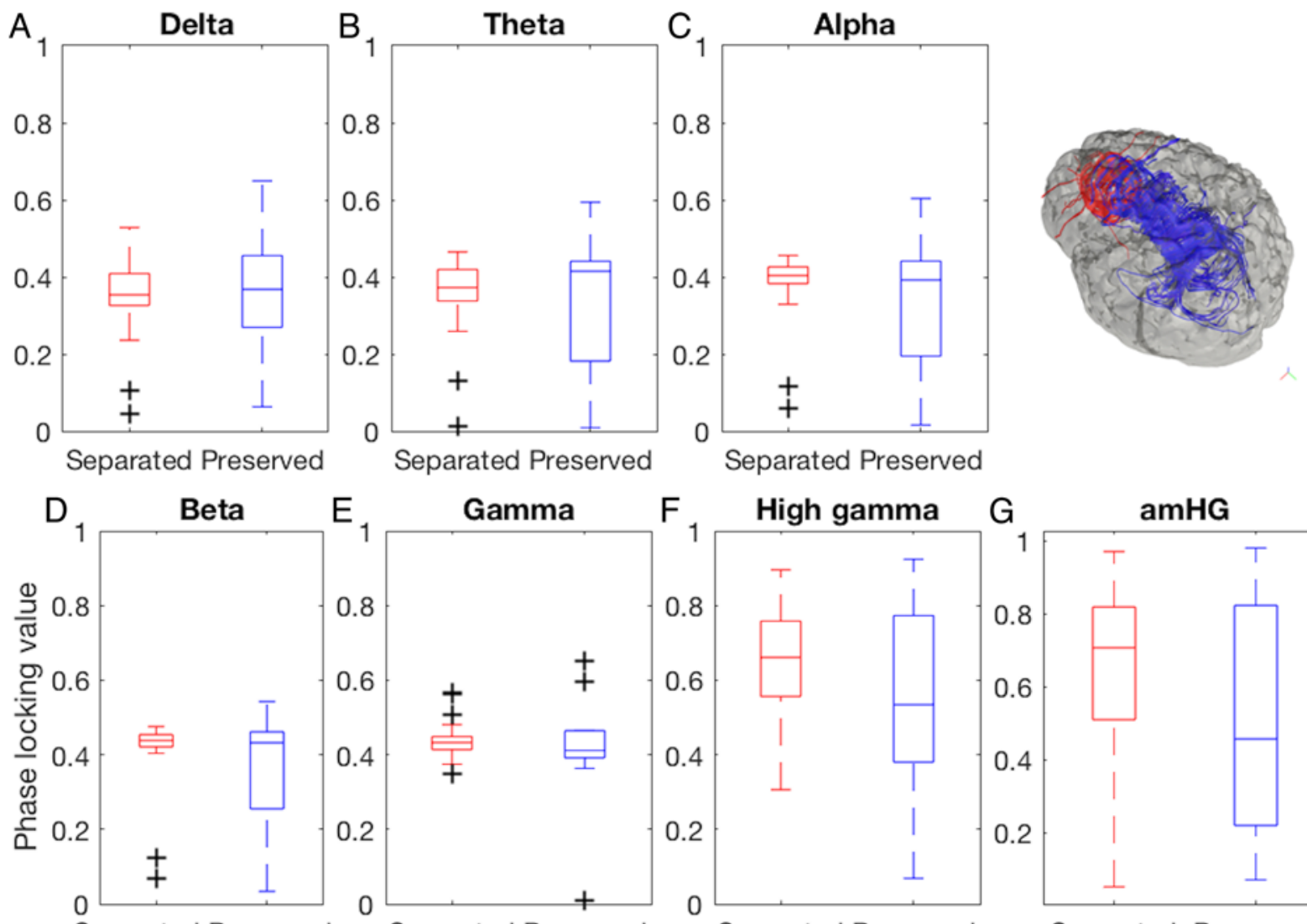

Separated Preserved
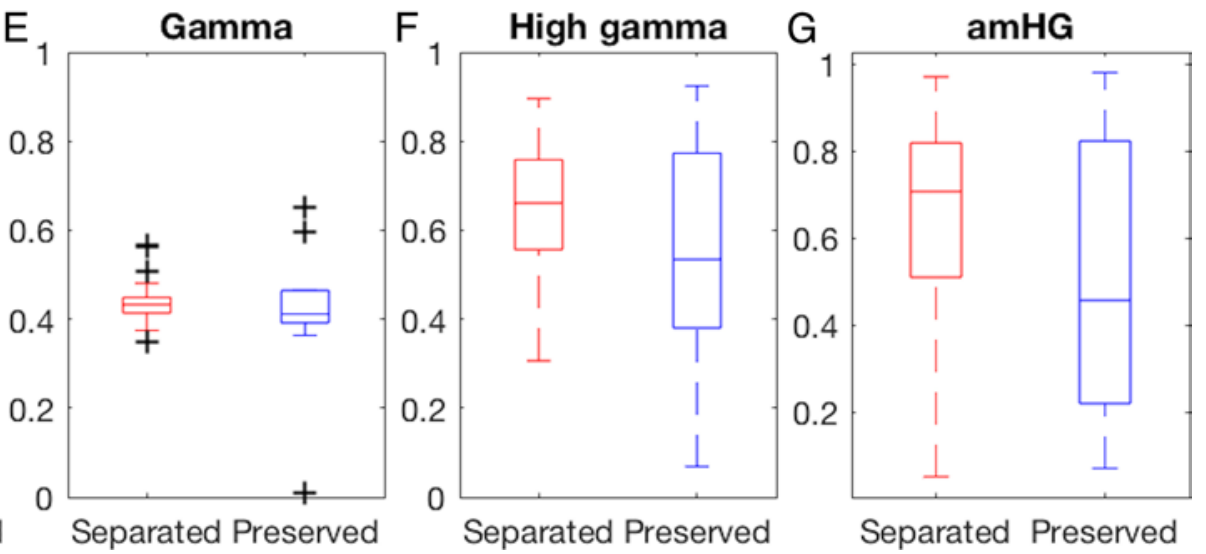

FIG. 2. Box plots showing distribution of the PLV in separated and preserved pairs of electrodes, where members of an electrode pair are located over equivalent locations in each hemisphere. Boxes indicate interquartile range and median, hash marks indicate most extreme non-outlier values, and plus signs indicate outliers. There were no significant differences in any comparison (all $p$ $>0.05)$ A: Delta $(0-4 \mathrm{~Hz}), p=0.79$. B: Theta $(4-8 \mathrm{~Hz}), p=0.61$. C: Alpha $(8-13 \mathrm{~Hz}), p=0.57$. D: Beta $(13-30 \mathrm{~Hz}), p=0.66$. E: Gamma $(30-70 \mathrm{~Hz}), p=0.36$. F: HG $(70-200 \mathrm{~Hz}), p=0.31$. G: amHG $(0.1-1 \mathrm{~Hz}$ modulations of amplitude in $70-$ to $200-\mathrm{Hz}$ range), $p=0.27$.

no relationship between the position of the electrode pair and connectivity strength in any of the frequency bands of interest (Fig. 3, $\mathrm{R}^{2}$ values). The higher median and larger spread in PLV in HG and amHG compared to the other bands was again visible here. We also observed that the outliers seen in the box plots can all be attributed to electrodes that were close to the disconnection point (i.e., the transition between blue and red markers).

\section{Discussion}

This report is the first demonstration of the nonacute (7 days postoperative) effect of a partial callosotomy on interhemispheric electrophysiological connectivity measured with ECoG. We found no significant differences in interhemispheric connectivity strength between regions whose direct corpus callosum connection was severed and regions whose connection was preserved.

Prior studies that involved postoperative MRI and scalp EEG or intraoperative (not long-term) ECoG investigation of synchrony after disconnection of the corpus callosum have found that synchrony is disrupted acutely ${ }^{1,6,14,21}$ but rebounds to typical or near-typical levels in the weeks to years after the procedure..$^{15,30,32}$ Variations in seizure se- verity and presentation, level of seizure disruption following surgery, and length of time between surgery and measurement of interhemispheric connectivity may explain disparities in prior results.

We propose several explanations for the observed maintenance of connectivity strength after partial corpus callosotomy, though our single case report cannot be widely generalized. The remaining corpus callosum may be compensating for the loss of the anterior portion, and the still-connected cortical regions may act to indirectly synchronize the now-separated areas of cortex. ${ }^{22}$ Prior work found elevated compensatory connectivity in the surviving portion of the corpus callosum in individuals with partial agenesis of the corpus callosum. ${ }^{28}$

Alternatively, other sources such as the thalamus may be providing inputs to both hemispheres, which then respond similarly to the common source, especially for low frequencies. If true, the observed synchrony would be more precisely described as the hemispheres synchronizing to a common source.22

This case study is limited to examination of separated versus preserved electrodes at a single time point. Due to the presence of the VNS, the anatomical MRI and DTI 

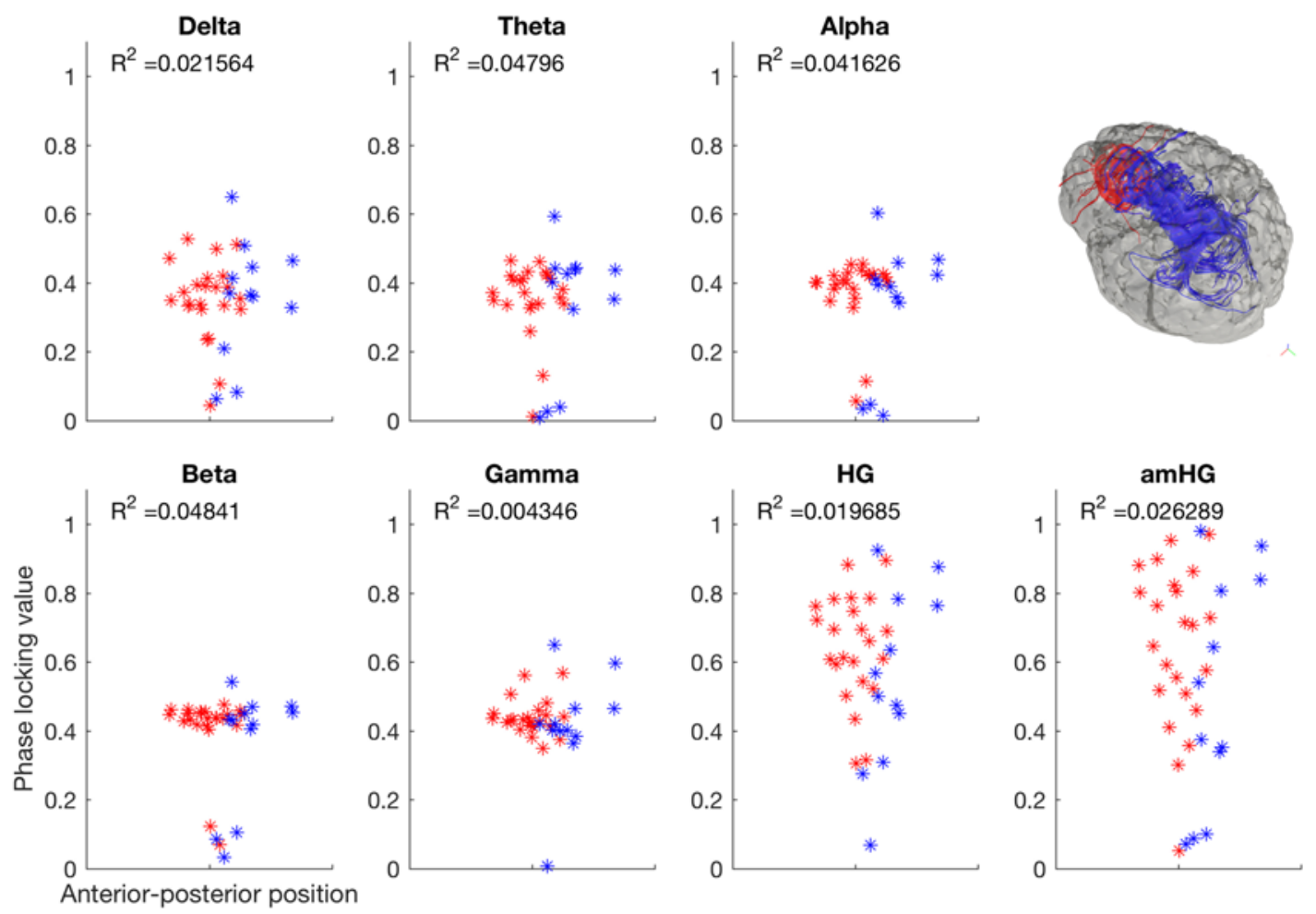

FIG. 3. Scatter plots showing distribution of PLV as a function of anterior-posterior position of pair, represented by the $Y$ coordinate of right hemisphere electrode for each pair. Red markers are separated pairs of electrodes; blue markers are preserved pairs. $R^{2}$ correlation coefficients of all pairs are marked on each plot. A: Delta $(0-4 \mathrm{~Hz})$. B: Theta $(4-8 \mathrm{~Hz})$. C: Alpha (8-13 Hz). D: Beta (13-30 Hz). E: Gamma (30-70 Hz). F: HG (70-200 Hz). G: amHG (0.1-1 Hz modulations of amplitude in 70- to 200-Hz range). The essentially identical distributions of PLVs are evident for electrodes within, or posterior to, the extent of the corpus callosum disconnection.

scans had relatively poor resolution, leading to greater uncertainty in the tract tracing and therefore the loss of a relatively large number of electrode pairs ( 9 pairs of 47 [19\%]) that we could not assign to the separated or preserved groups with confidence. Finally, this study is limited in that the data were obtained from a single case. Nevertheless, the absence of a strong alteration of connectivity is at odds with other findings, which did not control for epileptic activity, which, itself, may drive diminished connectivity. ${ }^{5}$ Further studies may compare the effect of a partial disconnection of the corpus callosum to a complete disconnection, which may result in different changes to interhemispheric connectivity. ${ }^{6}$

\section{Acknowledgments}

This work was supported by NSF grant EEC-1028725 for the Center for Sensorimotor Neural Engineering.

\section{References}

1. Brázdil M, Brichta J, Krajča V, Kuba R, Daniel P: Interhemispheric EEG coherence after corpus callosotomy. Eur J Neurol 4:419-425, 1997

2. Casimo K, Darvas F, Wander J, Ko A, Grabowski TJ, Novotny $\mathrm{E}$, et al: regional patterns of cortical phase synchrony in the resting state. Brain Connect 6:470-481, 2016

3. Gazzaniga MS: Forty-five years of split-brain research and still going strong. Nat Rev Neurosci 6:653-659, 2005
4. Hinkley LBN, Marco EJ, Findlay AM, Honma S, Jeremy RJ, Strominger Z, et al: The role of corpus callosum development in functional connectivity and cognitive processing. PLoS One 7:e39804, 2012

5. James GA, Tripathi SP, Ojemann JG, Gross RE, Drane DL: Diminished default mode network recruitment of the hippocampus and parahippocampus in temporal lobe epilepsy. $\mathbf{J}$ Neurosurg 119:288-300, 2013

6. Johnston JM, Vaishnavi SN, Smyth MD, Zhang D, He BJ, Zempel JM, et al: Loss of resting interhemispheric functional connectivity after complete section of the corpus callosum. J Neurosci 28:6453-6458, 2008

7. Keller CJ, Bickel S, Honey CJ, Groppe DM, Entz L, Craddock RC, et al: Neurophysiological investigation of spontaneous correlated and anticorrelated fluctuations of the BOLD signal. J Neurosci 33:6333-6342, 2013

8. Khanna PC, Poliakov AV, Ishak GE, Poliachik SL, Friedman $\mathrm{SD}$, Saneto RP, et al: Preserved interhemispheric functional connectivity in a case of corpus callosum agenesis. Neuroradiology 54:177-179, 2012

9. Ko AL, Darvas F, Poliakov A, Ojemann J, Sorensen LB: Quasi-periodic fluctuations in default mode network electrophysiology. J Neurosci 31:11728-11732, 2011

10. Ko AL, Weaver KE, Hakimian S, Ojemann JG: Identifying functional networks using endogenous connectivity in gamma band electrocorticography. Brain Connect 3:491-502, 2013

11. Lachaux JP, Rodriguez E, Martinerie J, Varela FJ: Measuring phase synchrony in brain signals. Hum Brain Mapp 8:194-208, 1999

12. Margulies DS, Kelly AMC, Uddin LQ, Biswal BB, Castella- 
nos FX, Milham MP: Mapping the functional connectivity of anterior cingulate cortex. Neuroimage 37:579-588, 2007

13. Nir Y, Mukamel R, Dinstein I, Privman E, Harel M, Fisch $\mathrm{L}$, et al: Interhemispheric correlations of slow spontaneous neuronal fluctuations revealed in human sensory cortex. Nat Neurosci 11:1100-1108, 2008

14. Okumura E, Iwasaki M, Sakuraba R, Itabashi I, Osawa S, Jin K, et al: Time-varying inter-hemispheric coherence during corpus callosotomy. Clin Neurophysiol 124:2091-2100, 2013

15. O'Reilly JX, Croxson PL, Jbabdi S, Sallet J, Noonan MP, Mars RB, et al: Causal effect of disconnection lesions on interhemispheric functional connectivity in rhesus monkeys. Proc Natl Acad Sci U S A 110:13982-13987, 2013

16. Paglioli E, Martins WA, Azambuja N, Portuguez M, Frigeri TM, Pinos L, et al: Selective posterior callosotomy for drop attacks: a new approach sparing prefrontal connectivity. Neurology 87:1968-1974, 2016

17. Papademetris X, Jackowski MP, Rajeevan N, DiStasio M, Okuda H, Constable RT, et al: Biolmage Suite: an integrated medical image analysis suite: an update. Insight J 2006:209, 2006

18. Poliachik SL, Poliakov AV, Jansen LA, McDaniel SS, Wray $\mathrm{CD}$, Kuratani J, et al: Tissue localization during resective epilepsy surgery. Neurosurg Focus 34(6):E8, 2013

19. Quigley M, Cordes D, Turski P, Moritz C, Haughton V, Seth $\mathrm{R}$, et al: Role of the corpus callosum in functional connectivity. AJNR Am J Neuroradiol 24:208-212, 2003

20. Quigley M, Cordes D, Wendt G, Turski P, Moritz C, Haughton V, et al: Effect of focal and nonfocal cerebral lesions on functional connectivity studied with MR imaging. AJNR Am J Neuroradiol 22:294-300, 2001

21. Rojas-Ramos OA, Ondarza R, Ramos-Loyo J, Trejo-Martínez D, Del Río-Portilla Y, Guevara MA, et al: Acute effect of callosotomy on cortical temporal coupling in humans: intraoperative electrocorticographic recording. Clin Neurophysiol 124:1959-1969, 2013

22. Roland JL, Snyder AZ, Hacker CD, Mitra A, Shimony JS, Limbrick DD, et al: On the role of the corpus callosum in interhemispheric functional connectivity in humans. Proc Natl Acad Sci U S A 114:13278-13283, 2017

23. Salvador R, Suckling J, Coleman MR, Pickard JD, Menon D, Bullmore E: Neurophysiological architecture of functional magnetic resonance images of human brain. Cereb Cortex 15:1332-1342, 2005

24. Simon-Dack SL, Holtgraves T, Hernandez K, Thomas C: Resting EEG and behavioural correlates of interhemispheric transfer times. Laterality 20:618-638, 2015

25. Stark DE, Margulies DS, Shehzad ZE, Reiss P, Kelly AMC,
Uddin LQ, et al: Regional variation in interhemispheric coordination of intrinsic hemodynamic fluctuations. J Neurosci 28:13754-13764, 2008

26. Tanriverdi T, Olivier A, Poulin N, Andermann F, Dubeau F: Long-term seizure outcome after corpus callosotomy: a retrospective analysis of 95 patients. J Neurosurg 110:332-342, 2009

27. Tognoli E, Kelso JA: Brain coordination dynamics: true and false faces of phase synchrony and metastability. Prog Neurobiol 87:31-40, 2009

28. Tovar-Moll F, Monteiro M, Andrade J, Bramati IE, ViannaBarbosa R, Marins T, et al: Structural and functional brain rewiring clarifies preserved interhemispheric transfer in humans born without the corpus callosum. Proc Natl Acad Sci U S A 111:7843-7848, 2014

29. Tyszka JM, Kennedy DP, Adolphs R, Paul LK: Intact bilateral resting-state networks in the absence of the corpus callosum. J Neurosci 31:15154-15162, 2011

30. Uddin LQ, Mooshagian E, Zaidel E, Scheres A, Margulies DS, Kelly AMC, et al: Residual functional connectivity in the split-brain revealed with resting-state functional MRI. Neuroreport 19:703-709, 2008

31. Wang D, Buckner RL, Liu H: Functional specialization in the human brain estimated by intrinsic hemispheric interaction. J Neurosci 34:12341-12352, 2014

32. Zhou IY, Liang YX, Chan RW, Gao PP, Cheng JS, Hu Y, et al: Brain resting-state functional MRI connectivity: morphological foundation and plasticity. Neuroimage 84:1-10, 2014

\section{Disclosures}

The authors report no conflict of interest concerning the materials or methods used in this study or the findings specified in this paper.

\section{Author Contributions}

Conception and design: Ojemann. Acquisition of data: Poliachik, Novotny, Poliakov, Ojemann. Analysis and interpretation of data: Casimo, Grassia, Poliachik. Drafting the article: Casimo. Critically revising the article: all authors. Reviewed submitted version of manuscript: Casimo, Ojemann. Approved the final version of the manuscript on behalf of all authors: Casimo. Statistical analysis: Casimo, Grassia. Study supervision: Ojemann.

\section{Correspondence}

Kaitlyn Casimo: University of Washington, Seattle, WA. kcasimo@uw.edu. 